\title{
Quantum Langevin equations for a two-mode parametric amplifier: Noise squeezing without negative diffusion
}

\author{
L. Sainz de los Terreros \\ Departamento de Matemática Aplicada, \\ Escuela Universitaria de Ingenieria Ténica Aeronaútica, Universidad Politécnica de Madrid, \\ Avenida Cardenal Cisneros 3, E-28040 Madrid, Spain \\ F. J. Bermejo \\ Instituto de Estructura de la Materia, Consejo Superior de Investigaciones Científicas, Serrano 119, E-28006 Madrid, Spain
}

(Received 20 May 1991)

\begin{abstract}
The theory of a two-mode nondegenerate parametric amplifier in a cavity is reformulated in terms of quadrature-phase-amplitude variables. The corrrespondence with a genuine classical stochastic linear process is found (non-negative-definite diffusion matrices) for the case of a cavity device immersed in thermal or ordinary (nonsqueezed) vacuum sources. A special kind of squeezing, i.e., quadrature squeezing [B. L. Schumaker, Phys. Rep. 135, 318 (1986)], is found to be characteristic of the internal steady state for the case of a cavity model subjected to an additional phase-sensitive noise source coupled to one of the two internal modes. Finally, the usual squeezing spectrum of the output field is calculated in both cases by means of an input-output formalism based upon a symmetric ordering scheme of noncommuting operators, well adapted for the quadrature-phase description of the fields involved in the interaction.
\end{abstract}

PACS number(s): 42.50.Dv, 42.50.Kb

\section{INTRODUCTION}

In the past few years there has been active research on the role of stochastic differential equations for the analysis of the reduction (squeezing) of noise in optical devices generating "nonclassical" states of the radiation field. More recently the related problem of the inputoutput relationships for quantized light emerging from resonant cavities has been the subject of several studies [1].

The reduction of quantum noise carried by one of the two conjugate quadrature components of the light field may be interpreted in terms of negative diffusion coefficients in a formally classical (c-number) set of Langevin-type equations. This set of equations which encompasses the quantum dynamics is obtained via a Fokker-Planck equation for the well-known quasiprobability $P$-distribution function, following the conventional steps of the quantum-classical correspondence theory [2-4], one of the cornerstones of quantum optics, and is extensively developed in the quantum theory of the laser and nonlinear-optical processes $[5,6]$.

This formalism makes use of the intracavity creationannihilation mode operators and their classical phasespace images (the mode-amplitude complex variables) as the basic variables. The failure to obtain positive-definite diffusion matrices does not diminish the usefulness of this class of stochastic equations: There is a well-established theoretical recipe, the phase-space representation theory based upon the so-called positive- $P$ distribution [7], by which the dynamics may be reformulated as a unique set of stochastic differential equations with positive diffusion coefficients in an extended double-dimension phase space. Moreover, a well-established albeit not intuitive recipe for the calculation of the output squeezing spectra in terms of the internal cavity spectrum has been developed, which rests upon a particular scheme of ordering (normal and time ordering) of two-time second-order correlation functions for the basic intracavity mode operators.

The positive- $P$ formalism is especially relevant when explicit nonlinearities are present in the equations of motion since the obtained nonlinear $c$-number Langevin equations are easier to handle than the equivalent quantum counterpart, i.e., the Heisenberg-Langevin operator equations.

It has long been emphasized [9] that the variables to be used for the description of quantum noise emerging from two-photon optical devices (i.e., parametric oscillators, two-photon lasers, etc.) should be the quadrature-phaseamplitude operators instead of the creation-annihilation photon operators. While the latter are tailored to account for one-photon optical devices, the former are the natural variables when the field consists of pairs of correlated photons.

In this paper we adopt this point of view as applied to linear (subthreshold) two-mode parametric amplifiers. The systematic use of quadrature-phase operators has a threefold advantage. First, the problem of negative diffusion does not appear in the classical correspondence. Second, and most important, this formalism gives a definite and simple recipe to calculate the out-of-thecavity squeezing spectrum, leading to a clear picture of the important problem of the relation between intracavity and output spectrum of noise. Third, the same recipe is used to account for squeezed input fields entering the cavity amplifier. Furthermore it might be automatically extended to other two-mode two-photon quantum-optical devices.

The paper is organized as follows. In Sec. II the nondegenerate two-mode parametric amplifier within the 
nondepleted pump approximation is considered. Since the problem to be considered is basically a linear one, the dynamics can be directly stated in the frame of linear quantum Heisenberg-Langevin equations for the quadrature-phase-amplitude operators. Two different kinds of coupling of the internal modes to the outside reservoir fields are considered: the usual ordinary vacuum or temperature-dependent thermal field and a phasesensitive reservoir [10], which may be used as a physical realization of a broadband squeezed multimode vacuum to which the cavity is eventually coupled through a second cavity mirror.

In Sec. III the isomorphism with a genuine classical stochastic process is discussed. The choosing of quadrature-phase amplitudes is shown to lead to wellbehaved (positive-definite) diffusion matrices.

Finally, in Sec. IV, the spectrum of squeezing is calculated outside the cavity by means of an input-output formalism tailored to the quadrature-phase physical picture of the process. Our main emphasis is the method itself. A well-defined scheme to calculate the out-of-the-cavity squeezing spectrum is proposed which is free from the difficulties associated with time-ordering problems present in the usual scheme of mode-amplitude operators. This provides also an intuitive and physically appealing way for the explanation of why full squeezing may be measured outside an ideal parametric amplifier in spite of the limited amount of intracavity squeezing.

Furthermore the same recipe allows the inclusion of broadband squeezed inputs and interesting results are obtained when it is applied to a cavity device coupled to a second broadband squeezed reservoir. The analysis shows that in this latter case, the output spectrum corresponds to an internal state with nonstationary quadrature-phase noise.

\section{HEISENBERG-LANGEVIN EQUATIONS FOR THE TWO-MODE QUADRATURE-PHASE AMPLITUDES}

\section{A. Cavity amplifier coupled to an ordinary vacuum field}

The model to be considered is a system of two resonant-cavity modes at frequencies $\Omega \pm v$, coupled by a nonlinear intracavity medium through the action of a pumping field considered as a classical coherent field at frequency $\Omega$. We write the model Hamiltonian as

$$
H=H_{s}+H_{b}+H_{s b}
$$

where

$$
\begin{aligned}
H_{s}=H_{0}+H^{\prime}(t)= & \hbar(\Omega+v) a_{+}^{\dagger} a_{+}+\hbar(\Omega-v) a_{-}^{\dagger} a_{-} \\
& +i \hbar g a_{+}^{\dagger} a_{-}^{\dagger} \exp (-2 i \Omega t)+\text { H.c. }
\end{aligned}
$$

is the free field plus the intermode coupling Hamiltonian. The free-bath and system-bath Hamiltonians are added as

$$
H_{b}=\sum_{\alpha} \hbar \omega_{1 \alpha} c_{1 \alpha}^{\dagger} c_{1 a}+\sum \hbar \omega_{2 \alpha} c_{2 \alpha}^{\dagger} c_{2 \alpha}
$$

and

$$
H_{s b}=\sum_{\alpha} \hbar k_{1 \alpha} c_{1 \alpha} a_{+}^{\dagger}+\sum_{\alpha} \hbar k_{2 \alpha} c_{2 \alpha} a_{-}^{\dagger}+\text { H.c. }
$$

where it has been assumed that the system modes decay into nonoverlapping distinct uncorrelated baths referred to by the subscripts 1 and 2, and hence it is required that either of the cavity linewidths are small compared with both the cavity spacing and the signal-idler detuning $v$.

Starting with the exact Heisenberg evolution equations for the system and bath modes, a set of HeisenbergLangevin quantum equations may be derived assuming that the correlation times of each of the independent baths to which the system modes are coupled are sufficiently short so that the interaction with the baths may be considered as Markovian.

The method to derive these equations is widely known (see, for example, Refs. [2-4], and references therein) and we do not repeat it here. The only point is that here we deal with two mutually interacting modes, with an interaction Hamiltonian quadratic in the mode creationannihilation operators, which is sufficient to ensure that the resulting Heisenberg-Langevin equations are linear. To be explicit we obtain

$$
\begin{aligned}
& \frac{d \widetilde{a}_{+}}{d t}=-\left(i v+\gamma_{+}\right) \widetilde{a}_{+}(t)+\gamma_{+} \widetilde{a}_{-}^{\dagger}(t)+\widetilde{\Gamma}_{+}(t), \\
& \frac{d \widetilde{a}_{-}}{d t}=\left(i v-\gamma_{-}\right) \widetilde{a}_{-}(t)+\gamma_{-} \widetilde{a}_{+}^{\dagger}(t)+\widetilde{\Gamma}_{-}(t)
\end{aligned}
$$

where $\gamma_{+}=\pi g\left(\omega_{+}\right)\left|k_{1}\left(\omega_{+}\right)\right|^{2}$ is the decay constant for the side mode at frequency $\omega_{+}=\Omega+v$, the density of bath modes around $\omega_{+}$being $g\left(\omega_{+}\right)$, and an analogous expression may be obtained for $\gamma_{-}$. The noise operators $\widetilde{\Gamma}_{ \pm}(t)$ may be formally written in terms of initial bath operators:

$$
\widetilde{\Gamma}_{+}(t)=-i \sum_{\alpha} k_{1 \alpha} \exp \left[-i\left(\omega_{1 \alpha}-\Omega\right) t\right] c_{1 \alpha}(0)
$$

and a similar expression may be written for $\widetilde{\Gamma}_{-}(t)$. Assuming a dense distribution of bath modes around the side-mode frequencies $\omega_{ \pm}=\Omega \pm v$, the correlation functions for these Langevin forces may be readily approximated as the well-known white-noise $\delta$-correlated functions [11]:

$$
\left\langle\widetilde{\Gamma}_{+}(t) \widetilde{\Gamma}_{-}\left(t^{\prime}\right)\right\rangle=\left\langle\widetilde{\Gamma}_{+}(t) \widetilde{\Gamma}_{+}\left(t^{\prime}\right)\right\rangle=\left\langle\widetilde{\Gamma}_{-}(t) \widetilde{\Gamma}_{-}\left(t^{\prime}\right)\right\rangle=0,
$$

$$
\left\langle\widetilde{\Gamma}_{ \pm}^{\dagger}\left(t^{\prime}\right) \widetilde{\Gamma}_{ \pm}(t)\right\rangle=\exp \left[-i v\left(t-t^{\prime}\right)\right] 2 \gamma_{ \pm} \bar{n}_{ \pm} \delta\left(t-t^{\prime}\right)
$$

$$
\left\langle\widetilde{\Gamma}_{ \pm}(t) \widetilde{\Gamma}_{ \pm}^{\dagger}\left(t^{\prime}\right)\right\rangle=\exp \left[-i v\left(t-t^{\prime}\right)\right] 2 \gamma_{ \pm}\left(1+\bar{n}_{ \pm}\right) \delta\left(t-t^{\prime}\right)
$$

where $\bar{n}_{ \pm}$denote the temperature-dependent spectral density of photons at the side-mode frequencies. Here we assume a reservoir field in an ordinary vacuum or thermal state coupled to the cavity through a single transmitting mirror, the photons that escape away from 
the cavity being the only source of losses for the internal field.

Without loss of generality we can assume that both decay constants are equal: $\gamma_{+}=\gamma_{-}$. The HeisenbergLangevin equations [Eqs. (2.5a) and (2.5b)] have been written in terms of the slowly varying operators $\widetilde{a}_{ \pm}(t)=\exp (i \Omega t) a_{ \pm}(t)$, which eliminates the highfrequency oscillation at the "carrier" frequency $\Omega$. We may then rewrite these equations in terms of the slowly varying quadrature-phase amplitudes (relative to the oscillation at "modulation" frequency $v$ ) [9]:

$Z_{1}=\widetilde{Z}_{1}(t) \exp (i v t)=\left[\lambda_{+} \widetilde{a}_{+}(t)+\lambda_{-} \widetilde{a}_{-}^{\dagger}(t)\right] \exp (i v t)$,

$$
\begin{aligned}
Z_{2} & =\widetilde{Z}_{2}(t) \exp (i v t) \\
& =\left[-i \lambda_{+} \widetilde{a}_{+}(t)+i \lambda_{-} \widetilde{a}_{-}^{\dagger}(t)\right] \exp (i v t)
\end{aligned}
$$

where $\lambda_{ \pm}=\sqrt{(\Omega \pm v) / 2 \Omega}$. Although the constants $\lambda_{ \pm}$ deserve some discussion (in connection with the definition and experimental relevance of quadrature-phase amplitudes $[9,10])$, we will, for the sake of clarity, maintain at the beginning the exact formulas that follow from this definition and, eventually, the approximation $v / \Omega \approx 0$ will be systematically adopted (see Sec. IV), which is valid for modulation frequency displacements $v$ of the cavity modes far below the optical range.

It is easy to transform the above Heisenberg-Langevin equations into the following ones, involving the quadrature-phase-amplitude operators:

$$
\begin{aligned}
& \frac{d Z_{1}}{d t}=-\gamma(1-\sigma) Z_{1}-i \gamma \sigma(v / \Omega) Z_{2}+\Gamma_{1}(t), \\
& \frac{d Z_{2}}{d t}=-\gamma(1+\sigma) Z_{2}-i \gamma \sigma(v / \Omega) Z_{1}+\Gamma_{2}(t)
\end{aligned}
$$

where $\sigma=(g / \gamma)\left[1-(v / \Omega)^{2}\right]^{-1 / 2}$ is the pumping parameter, and $g$ has been assumed to be real for reasons of simplicity.

Taking into account Eqs. (2.6a)-(2.6c) with $\bar{n}_{+}$ $=\bar{n}_{-}=\bar{n}$, the transformed noise operators $\Gamma_{i}(t)(i=1,2)$ are found to have zero averages, and correlation functions are given by

$$
\begin{aligned}
\left\langle\Gamma_{2}^{\dagger}\left(t^{\prime}\right) \Gamma_{1}(t)\right. & =-\left\langle\Gamma_{1}^{\dagger}\left(t^{\prime}\right) \Gamma_{2}(t)\right\rangle \\
& =-i \gamma[1-(v / \Omega)(1+2 \bar{n})] \delta\left(t-t^{\prime}\right), \\
\left\langle\Gamma_{1}(t) \Gamma_{2}^{\dagger}\left(t^{\prime}\right)\right. & =-\left\langle\Gamma_{2}(t) \Gamma_{1}^{\dagger}\left(t^{\prime}\right)\right\rangle \\
& =i \gamma[1+(v / \Omega)(1+2 \bar{n})] \delta\left(t-t^{\prime}\right), \\
\left\langle\Gamma_{2}^{\dagger}\left(t^{\prime}\right) \Gamma_{2}(t)\right\rangle & =\left\langle\Gamma_{1}^{\dagger}\left(t^{\prime}\right) \Gamma_{1}(t)\right\rangle \\
& =\gamma[(1+2 \bar{n})-(v / \Omega)] \delta\left(t-t^{\prime}\right), \\
\left\langle\Gamma_{2}(t) \Gamma_{2}^{\dagger}\left(t^{\prime}\right)\right\rangle & =\left\langle\Gamma_{1}^{\dagger}(t) \Gamma_{1}\left(t^{\prime}\right)\right\rangle \\
& =\gamma[(1+2 \bar{n})+(v / \Omega)] \delta\left(t-t^{\prime}\right), \\
\left\langle\Gamma_{i}(t) \Gamma_{k}\left(t^{\prime}\right)\right\rangle & =0 .
\end{aligned}
$$

We note in turn that a $2 \times 2$ complex diffusion matrix may be defined by

$$
\left\langle\Gamma_{i}(t) \Gamma_{k}^{\dagger}\left(t^{\prime}\right)\right\rangle_{\mathrm{sym}}=\mathcal{D}_{i k} \delta\left(t-t^{\prime}\right)
$$

(where the "sym" denotes symmetric averages), and therefore becomes the following Hermitian matrix:

$\mathcal{D}_{s}=\left(\begin{array}{cc}\gamma(1+2 \bar{n}) & i \gamma(v / \Omega)(1+2 \bar{n}) \\ -i \gamma(\nu / \Omega)(1+2 \bar{n}) & \gamma(1+2 \bar{n})\end{array}\right)$,

that is, a positive-definite Hermitian matrix, with positive eigenvalues:

$$
\lambda=\gamma(1+2 \bar{n})(1 \pm v / \Omega) .
$$

It may also be easily proved [11] that these correlation functions just ensure the time invariance of the equal time commutator algebra for the quadrature-phase amplitudes:

$$
\begin{aligned}
& {\left[Z_{1}(t), Z_{2}^{\dagger}(t)\right]=\left[Z_{1}^{\dagger}(t), Z_{2}(t)\right]=i,} \\
& {\left[Z_{1}(t), Z_{1}^{\dagger}(t)\right]=\left[Z_{2}(t), Z_{2}^{\dagger}(t)\right]=v / \Omega,} \\
& {\left[Z_{k}(t), Z_{i}(t)\right]=0 .}
\end{aligned}
$$

Some comments about Eqs. (2.8a) and (2.8b) are in order. First of all it has to be taken into account that because of operator ordering, the change in the picture from mode-amplitude operators $a_{ \pm}$to quadrature-phaseamplitude operators $Z_{1,2}$ does not only involve a linear change of variables. In fact the Heisenberg-Langevin equations (2.8a) and (2.8b) will be shown in the next section to be entirely equivalent to a classical OrnsteinUhlenbeck process for two coupled complex variables (which are the classical $c$-number counterparts of the quadrature-phase-amplitude operators), once a definite (i.e., the symmetric) ordering scheme (relative to quadrature operators) is adopted.

The key point is that, when we adopt such a scheme, a classical stochastic picture emerges for the quantum process (see Sec. III): intermode parametric gain (modeled with the coupling constant $g$ ), associated with the semiclassical deterministic dynamics tends to redistribute the vacuum quadrature noise between orthogonal quadrature components, while the noise source appears in the classical Langevin equations just as it does in the quantum description, that is, it depends entirely upon the vacuum fluctuations of the bath and not on the coupling constant $g$.

It may now be understood, at least in part, why the usual formalism based upon mode-amplitude variables fails to allow a physical description in terms of a classical diffusion stochastic process: the intracavity coupling between modes $a_{ \pm}$develops purely quantum intensity cross correlations between them that eventually (once the steady state is reached) becomes stronger than individual self-correlations into each mode separately. This frame is thus suited to a photon-correlation view of two-mode squeezing but is entirely foreign to a phase-space description in terms of $c$-number classical amplitudes: in fact, no classical positive diffusion (with a source of real noise) process describing a two-mode coupling process between complex-mode field amplitudes may violate the classical Schwarz inequality [12]. No equivalent violation is apparent when quadrature-phase-amplitude variables are 
taken at the basis of the physical description and therefore this allows us to infer that quantum squeezing in this linear device (below threshold parametric oscillator) is essentially similar to the "classical squeezing" of noise recently reported [13].

\section{B. Amplifier coupled}

to a second phase-sensitive reservoir

The role of phase-sensitive reservoirs has been recognized recently to be important in connection with the behavior of quantum-optical systems in a broadband squeezed-vacuum environment $[14,15]$. Phase-dependent reservoirs give rise, as opposed to a broadband ordinary multimode vacuum, to some additional phase-dependent correlation functions that reflect the nonstationary and nonuniform, phase-dependent distribution of the noise in the reservoir.

Here, we consider two resonant modes of a high- $Q$ cavity in a ring configuration. The coupling to a squeezed reservoir field is now modeled to resolve a definite physical problem: a nondegenerate parametric amplification process occurs inside the cavity with two mirrors coupling the cavity to the outside world: the first mirror (see Fig. 1) transmits photons at both of the cavity resonant frequencies into an ordinary vacuum environment and is assumed to be the port used for the measurement of the emerging squeezed field while the second is fed by a broadband squeezed-vacuum field centered at one of the two internal frequencies. It is supposed, furthermore, that mirror $D$ is frequency selective in its transmission coefficient, allowing the leakage of photons only in the frequency band around the lower cavity resonance $\omega_{-}$.

As the loss through any unused port is well known to degrade the maximum obtainable squeezing, it is interesting to answer quantitatively how much the proposed device would compensate this undesired effect.

We assume therefore that there are now present two kinds of out-cavity reservoir field modes (operators $c$ and $d$ refer, respectively, to the mode operators of the quantized reservoir vacuum fields reflecting at mirrors $C$ and $D$ in Fig. 1), so that the relaxation associated with the transmitting mirrors $C$ and $D$ is written

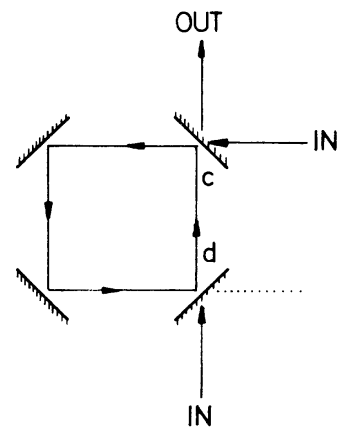

FIG. 1. Schematic representation of the device considered here. The phase-insensitive vacuum fluctuations are considered to enter the cavity through mirror $c$ and the output spectrum of squeezing is measured at a right angle. The mirror $d$ enables the entrance of phase-sensitive squeezed input noise depending upon the parameter $r$ and the transmittivity ratio $q$.

$$
\begin{aligned}
H_{s b}= & \sum_{\alpha} \hbar k_{1 \alpha} c_{1 \alpha} a_{+}^{\dagger}+\sum_{\alpha} \hbar k_{2 \alpha} c_{2 \alpha} a_{-}^{\dagger} \\
& +\sum_{\alpha} \hbar k_{3 \alpha} d_{3 \alpha} a_{-}^{\dagger}+\text { H.c. }
\end{aligned}
$$

where now the third term models the losses of one of the intracavity modes (frequency $\omega_{-}$), through the coupling mirror $D$. The frequency band over which the last sum in Eq. (2.14) is considered is assumed to correspond to a broadband squeezed-vacuum field at the central frequency $\omega-=\Omega-v$. The exact Heisenberg equations for the intracavity modes may now be translated into approximate Heisenberg-Langevin operator equations as above. The result is now formally the same as the one obtained in Sec. II A (there the intracavity photons leaked into a multimode out-cavity field in an ordinary vacuum state through a single port cavity):

$$
\begin{aligned}
& \frac{d \widetilde{a}_{+}}{d t}=-\left(i v+\gamma_{+}\right) \widetilde{a}_{+}(t)+g \widetilde{a}_{-}^{\dagger}(t)+\widetilde{\Gamma}_{+}(t), \\
& \frac{d \widetilde{a}_{-}}{d t}=\left(i v-\gamma_{-}\right) \widetilde{a}_{-}(t)+g \widetilde{a}_{+}^{\dagger}(t)+\widetilde{\Gamma}_{-}(t) .
\end{aligned}
$$

We assume equal transmission at mirror $C$ for both intracavity modes $\left(\gamma_{c_{-}}=\gamma_{c_{+}}\right)$, i.e., $\gamma_{+}=\gamma_{c_{+}}$ $=\pi g\left(\omega_{+}\right)\left|k_{1}\left(\omega_{+}\right)\right|^{2}, \quad \gamma_{-}=\gamma_{c_{-}}+\gamma_{d_{-}} \quad$ where $\gamma_{c_{-}}=\pi g\left(\omega_{-}\right)\left|k_{2}\left(\omega_{-}\right)\right|^{2}$, and $\gamma_{d_{-}}=\pi g\left(\omega_{-}\right)\left|k_{3}\left(\omega_{-}\right)\right|^{2}$. We shall write, for brevity, $\gamma_{+}=\gamma$, and $\gamma_{-}=(1+q) \gamma$, where $q$ is the ratio of the decaying rates at the lower resonant frequency $\omega_{-}$for the two mirrors $\left[q=\left(\gamma_{-}-\gamma_{+}\right) / \gamma_{+}=\gamma_{d_{-}} / \gamma_{c_{-}}\right]$.

The correlation functions for the Langevin forces in Eqs. (2.15a) and (2.15b) may be easily related to those of the reservoir fields. Assuming that only a narrow distribution of bath-mode frequencies centered around each of the resonant-cavity mode frequencies $\omega_{ \pm}=\Omega \pm v$ couples to the cavity, the noise operators

$$
\begin{aligned}
\widetilde{\Gamma}_{+}(t)= & -\sum_{\alpha} k_{1 \alpha} \exp \left[-i\left(\omega_{1 \alpha}-\Omega\right) t\right] c_{1 \alpha}(0), \\
\widetilde{\Gamma}_{-}(t)= & -i \sum_{\alpha} k_{2 \alpha} \exp \left[-i\left(\omega_{2 \alpha}-\Omega\right) t\right] c_{2 \alpha}(0) \\
& -i \sum_{\alpha} k_{3 \alpha} \exp \left[-i\left(\omega_{3 \alpha}-\Omega\right) t\right] d_{3 \alpha}(0)
\end{aligned}
$$

may be approximated as [17]

$$
\begin{aligned}
\widetilde{\Gamma}_{+}(t)= & \widetilde{\Gamma}_{c_{+}}(t)=-i \exp \left(i \zeta_{c+}\right)(1+v / \Omega)^{-1 / 2} \\
& \times \sqrt{2 \gamma} \widetilde{\mathscr{E}}_{c_{+}}^{(+)}(t) \\
\widetilde{\Gamma}_{-}(t)= & \widetilde{\Gamma}_{c_{-}}(t)+\widetilde{\Gamma}_{d_{-}}(t) \\
= & -i \exp \left(i \zeta_{c_{-}}\right)(1-v / \Omega)^{-1 / 2} \sqrt{2 \gamma} \widetilde{\mathscr{E}}_{c_{-}}^{(+)}(t) \\
& -i \exp \left(i \zeta_{d_{-}}\right) \sqrt{2 q \gamma} \widetilde{\mathscr{E}}_{d_{-}}^{(+)}(t)
\end{aligned}
$$

where the complex exponentials are related to the phase shifts due to transmission and

$\widetilde{\mathscr{E}}_{c_{+}}^{(+)}(t)=\left[\frac{2 \epsilon_{0} c A}{\hbar \Omega}\right]^{1 / 2} \exp (i \Omega t) E_{c_{+}}^{(+)}(t)$, 
$\widetilde{\mathscr{E}}_{c_{-}}^{(+)}(t)=\left(\frac{2 \epsilon_{0} c A}{\hbar \Omega}\right)^{1 / 2} \exp (i \Omega t) E_{c_{-}}^{(+)}(t)$

$\mathscr{E}_{d_{-}}^{(+)}(t)=\left(\frac{2 \epsilon_{0} c A}{\hbar(\Omega-v)}\right)^{1 / 2} \exp (i \Omega t) E_{d_{-}^{(+)}}^{(t)}$,

etc. Notice that the factors $(1 \pm v / \Omega)^{-1 / 2}$ in Eqs. (2.17a) and (2.17b) appear as the result of the normalization adopted for the corresponding field operators $\widetilde{E}^{(+)}$. Here we have denoted also by $E_{c_{+}}^{(+)}(t)$ the free evolving positive-frequency part of the electric field operator of the corresponding reservoir field [analogous expressions may be written for $E_{c_{-}}^{(+)}(t)$ and $\left.E_{d_{-}}^{(+)}(t)\right]$ :

$$
E_{c_{+}}^{(+)}(t)=\sum_{\alpha}\left[\frac{\hbar \omega_{1 \alpha}}{2 \epsilon_{0} A L}\right] \exp \left(-i \omega_{1 \alpha} t\right) c_{1 \alpha}(0) .
$$

( $A$ is an effective cross-section area and $L$ is the length along the quantization coordinate; see the work of Carmichael in [21] for the details of the quantization scheme.)

In a similar fashion the other Langevin forces may be easily related to the reservoir field operators evolving freely from their value at the initial time. In doing so, the corresponding correlation functions are immediately obtained by averaging over the respective initial states of the reservoirs. We assume also without loss of generality that all the phase shifts are set equal to $\exp (-\pi / 2)$.

If the bandwidth of the squeezed reservoir field incoming through the mirror $D$ is much greater than the cavity linewidth, the vacuum quantum fluctuations appear as $d$ correlated squeezed white noise [17] to the cavity modes. For the reservoir in an ideal squeezed-vacuum state, the following correlation functions may be written [9]:

$$
\begin{aligned}
\left\langle\widetilde{\mathscr{E}}_{d_{-}}^{(+)}(t) \widetilde{\mathscr{E}}_{d_{-}}^{(+)}\left(t^{\prime}\right)\right\rangle & =\left\langle\widetilde{\mathscr{E}}_{d_{-}}^{(-)}(t) \widetilde{\mathscr{E}}_{d_{-}}^{(-)}\left(t^{\prime}\right)\right\rangle^{*} \\
& =-S^{(+)}(0) \exp \left[i v\left(t+t^{\prime}\right)\right] d\left(t-t^{\prime}\right),
\end{aligned}
$$

$$
\begin{aligned}
\left\langle\widetilde{\mathscr{E}}_{d_{-}}^{(+)}(t) \widetilde{\mathscr{E}}_{d_{-}}^{(-)}\left(t^{\prime}\right)\right\rangle= & \frac{1}{2}\left[1+\Sigma^{(+)}(0)+\Sigma^{(-)}(0)\right] \\
& \times \exp \left[i v\left(t-t^{\prime}\right)\right] d\left(t-t^{\prime}\right), \\
\left\langle\widetilde{E}_{d_{-}}^{(-)}(t) \widetilde{E}_{d_{-}^{(+)}}^{\left.\left(t^{\prime}\right)\right\rangle=}\right. & \frac{1}{2}\left[-1+\Sigma^{(+)}(0)+\Sigma^{(-)}(0)\right] \\
& \times \exp \left[-i v\left(t-t^{\prime}\right)\right] d\left(t-t^{\prime}\right)
\end{aligned}
$$

where the corresponding spectral densities (cf. Ref. [9])

$$
\begin{aligned}
\Sigma^{( \pm)}(\epsilon)=\frac{1}{2} & {\left[\left(\omega_{-} \pm \epsilon\right) / \omega_{-}\right] } \\
& \times\left\{\cosh ^{2}[r(\epsilon)]+\sinh ^{2}[r(\epsilon)]\right\}, \\
S^{( \pm)}(\epsilon)=- & {\left[\left(\omega_{-} \pm \epsilon\right) / \omega_{-}\right] } \\
& \times\left\{\cosh [r(\epsilon)] \sinh [r(\epsilon)] e^{ \pm 2 i \phi(\epsilon)}\right\}
\end{aligned}
$$

are replaced in Eqs. (2.19a)-(2.19c) by their values at the center of the broadband squeezed spectrum $(\epsilon=0)$. In this formulas $r$ and $\phi$ are parameters that characterize the squeezing.
We transform the Langevin equations [Eqs. (2.5a) and (2.5b)] writing them again in terms of quadrature-phaseamplitude operators as defined above [Eqs. (2.7a) and (2.7b)]. Using Eqs. (2.17) and (2.19)-(2.21), we arrive at the following Heisenberg-Langevin equations:

$$
\begin{aligned}
\left(\frac{d}{d t}+i v\right) \widetilde{Z}_{1}(t)= & -\gamma\left(1+\frac{q}{2}\right) \widetilde{Z}_{1}+\sigma \gamma \sqrt{1+q} \widetilde{Z}_{1} \\
& +\frac{1}{2} i \gamma q \widetilde{Z}_{2}+\widetilde{\Gamma}_{1}(t) \\
\left(\frac{d}{d t}+i v\right) \widetilde{Z}_{2}(t)= & -\gamma\left(1+\frac{q}{2}\right) \widetilde{Z}_{2}-\sigma \gamma \sqrt{1+q} \widetilde{Z}_{2} \\
& -\frac{1}{2} i \gamma q \widetilde{Z}_{1}+\widetilde{\Gamma}_{2}(t)
\end{aligned}
$$

where we have introduced again the pump parameter $\sigma=g / g_{t h}=g / \gamma \sqrt{1+q}$. In these and subsequent equations we shall use the approximation $v / \Omega \approx 0$.

From Eqs. (2.17) the noise operators $\widetilde{\Gamma}_{k}(t)$ may be related to the reservoir fields by

$\widetilde{\Gamma}_{1}(t)=-\sqrt{\gamma}\left[\widetilde{\mathscr{E}}_{c_{+}}^{(+)}(t)+\widetilde{\mathscr{E}}_{c_{+}}^{(-)}(t)\right]-\sqrt{q \gamma} \widetilde{\mathscr{E}}_{d_{-}}^{(-)}(t)$,

$\widetilde{\Gamma}_{2}(t)=-\sqrt{\gamma}\left[-i \widetilde{\mathscr{E}}_{c_{+}}^{(+)}(t)+i \widetilde{\mathscr{E}}_{c_{+}}^{(-)}(t)\right]-i \sqrt{q \gamma} \widetilde{\mathscr{E}}_{d_{-}}^{(+)}(t)$

$\left(\widetilde{\mathscr{E}}_{k}^{(-)}(t)=\left[\widetilde{\mathscr{E}}_{k}^{(+)}(t)\right]^{\dagger}, k=c_{ \pm}, d_{-}\right)$. Making use of Eqs. $(2.19 a)-(2.19 c)$ we readily arrive at the desired correlated functions. For convenience at a later step, we arrange the noise-operator variables in terms of the vector $\Gamma(t)=\left(\Gamma_{1}, \Gamma_{2}, \Gamma_{1}^{\dagger}, \Gamma_{2}^{\dagger}\right)^{T}$ and drop the time dependence at the modulation frequency $v\left[\Gamma_{k}(t)=\widetilde{\Gamma}_{k}(t) \exp (i v t)\right]$. We may then write

$$
\left\langle\Gamma(t) \Gamma\left(t^{\prime}\right)^{T}\right\rangle=\underline{D} d\left(t-t^{\prime}\right) .
$$

Taking into account Eqs. (2.19)-(2.23), the following $4 \times 4$ diffusion matrix results:

$$
\underline{D}=\underline{D}^{\mathrm{nsq}}+\underline{D}^{\mathrm{sq}}
$$

where the symbols "nsq" and "sq" denote the nonsqueezed and squeezed inputs, respectively:

$\underline{D}^{\mathrm{sq}}=\gamma\left(\begin{array}{cccc}0 & 0 & 1 & i \\ 0 & 0 & -i & 1 \\ 1 & i & 0 & 0 \\ -i & 1 & 0 & 0\end{array}\right)$,

$\underline{D}^{\mathrm{sq}}=q \gamma\left(\begin{array}{cccc}M e^{-2 i \phi} & i M e^{-2 i \phi} & N & -i N \\ i M e^{-2 i \phi} & -M e^{-2 i \phi} & i N & N \\ 1+N & i[1+N] & M e^{2 i \phi} & -i M e^{-2 i \phi} \\ -i[1+N] & 1+N & -i M e^{2 i \phi} & -M e^{2 i \phi}\end{array}\right)$

where $N=\sinh ^{2}(r), M=\sinh (r) \cosh (r)$. Notice that obviously the previous drift and diffusion matrices are recovered for $q=0$. The phase-sensitive diffusion matrix $\underline{D}^{\text {sq }}$ is expected to modify the spectrum of quadrature 
fluctuations of the field emerging from the cavity and further discussion is deferred to Sec. IV.

In connection with the present work it should be mentioned that the relaxation or decay of a cavity two-mode system interacting with a phase-sensitive reservoir has recently been studied by Ekert and Knight [16], by means of master-equation techniques.

\section{EQUIVALENCE TO A CLASSICAL PROCESS}

\section{A. Classical $c$-number Langevin equations}

The equivalence between quantum HeisenbergLangevin equations and ordinary classical diffusion processes is not a trivial question even if the former are linear in form. In fact, it is not self-evident how linear Langevin operator equations, like the ones obtained above [see Eqs. (2.8a) and (2.8b) and (2.22a) and (2.22b)], might be transformed into the corresponding $c$-number Langevin equations with equivalent positive-definite diffusion matrices. As is immediately recognized, problems associated with the ordering of noncommuting operators appear soon.

Recent efforts have been directed at describing quantum squeezing in a more classical manner. Marshall and Santos [8] have shown how c-number classical Langevin equations may be readily obtained from the Heisenberg equations for a typical squeezed field generator (the parametric oscillator), provided that, as usual, the model is treated within the linear approximation.

The main features of the method referred to above are (a) the role of bath modes is restored in the physical description as providing the proper source of noise (vacuum or thermal noise), (b) the coupling constants leading to squeezing are correctly assigned only to the deterministic (drift) terms of the Langevin equations, leaving the noise source (diffusion) generated by the dissipative interaction with the environment, and (c) the particular role of the Wigner function is emphasized as opposed to other phase-space representations.

The present formalism of a two-mode cavity amplifier, based upon the use of quadrature-phase amplitudes, enables us to achieve this correspondence: The quantum Langevin Eqs. (2.8a) and (2.8b) are in fact equivalent to classical $c$-number Langevin equations with ordinary white-noise sources. We limit ourselves here to the key arguments, leaving a complete account of the proof to a forthcoming paper. As Marshall and Santos [8] have pointed out in connection with the degenerate parametric amplifier, a system with at most quadratic reversible interaction Hamiltonian and weakly coupled to a set of densely distributed reservoir of harmonic oscillators evolves according to a classical stochastic system represented by the time-dependent Wigner distribution function.

As is well known, this is the phase-space image of the operator density matrix of the system, once the symmetric ordering of creation-annihilation variables is assumed. In our present two-mode situation we may notice by quick inspection that the normal (antinormal) order- ing of quadrature-phase amplitudes [see Eq. (3.8) below] corresponds to opposite ordering of the two modes in the single-mode sense (cf. Ref. [9]). Also the symmetric ordering of quadrature-phase amplitudes corresponds again to the same symmetric ordering scheme for the creationannihilation mode variables. We should expect, therefore, that a classical stochastic picture of the dynamics might be found in terms of $c$-number quadrature variables, governed by a corresponding two-mode Wigner function [18].

This is the first piece of our argument; the second, and most important, evolves from the form of the Langevin operator equations. As may be seen from Eqs. (2.8a) and (2.8b), the equations of motion have a block-diagonal drift matrix: the time evolution of quadrature-phase variables $\left(\widetilde{Z}_{k}\right)$ is decoupled from the corresponding to the Hermitian conjugates $\left(\widetilde{Z}_{m}^{\dagger}\right)$. This fact, together with the particular algebra of noncommuting quadrature-phaseamplitude operators, is sufficient to overcome the difficulties associated with time ordering in the passage from quantum to stochastic multitime correlation functions [19]. For brevity we limit ourselves to the case of a reservoir in an ordinary or thermal vacuum state (Sec. II A). We may thus write the following classical Langevin equations for $c$-number complex quadrature amplitudes:

$$
\begin{aligned}
& \frac{d \alpha_{1}}{d t}=-\gamma(1-\sigma) \alpha_{1}-i \gamma \sigma(v / \Omega) \alpha_{2}+\xi_{1}(t), \\
& \frac{d \alpha_{2}}{d t}=-\gamma(1+\sigma) \alpha_{2}-i \gamma \sigma(v / \Omega) \alpha_{1}+\xi_{2}(t)
\end{aligned}
$$

where $\xi_{i}(t)$ are now classical white-noise sources with identical correlations as the symmetric reservoir correlation functions [cf. Eq. (2.11)]:

$$
\begin{aligned}
& \left\langle\xi_{i}(t) \xi_{i}\left(t^{\prime}\right)^{*}\right\rangle_{\mathrm{sym}}=\gamma(1+2 \bar{n}) \delta\left(t-t^{\prime}\right) \quad(i=1,2), \\
& \left\langle\xi_{1}(t) \xi_{2}\left(t^{\prime}\right)^{*}\right\rangle_{\mathrm{sym}}=i \gamma(v / \Omega)(1+2 \bar{n}) \delta\left(t-t^{\prime}\right) .
\end{aligned}
$$

These correlations are evidently related to just the same positive-definite diffusion matrix defined above [Eq. (2.11)]. Beginning with these Langevin equations all symmetrized covariances and correlation functions may be derived to obtain exactly the same results that we would obtain by direct integration of the original quantum Langevin equations. The only effect of the parametric coupling between intracavity modes is to modify the effective damping constants of the two orthogonal quadratures [see Eq. (3.1)], thus producing a redistribution of vacuum fluctuations in each quadrature component of the two-mode field, i.e., squeezing.

A rigorous proof of the above statement may be established by means of formal phase-space methods as proposed earlier [20], and is planed to be the subject of a following paper. Here we notice that a certain FokkerPlanck equation may be written for an ideal two-photon device coupled to a heat or squeezed reservoir, starting from the master equation of the process, for a set of properly defined quasiprobability distribution functions (including the two-mode Wigner distribution).

The crucial properties of the normal (antinormal) or 
symmetric quadrature-phase ordering schemes involved in that formalism are that the diffusion matrices in this Fokker-Planck equation are just the same as the ones involved in the present quantum-operator Langevin treatment: as such, these diffusion matrices are positive definite, independent of the parametric pumping parameter $\sigma$, and reflect only the noise properties of the reservoir.

As it may be recognized, this physical picture is entirely different from (although equivalent to) the widely used phase-space representation based upon normal ordering of mode-amplitude operators, where an equivalent methodology leads to negative "nonclassical" diffusion.

\section{B. Generalized Einstein relations and quadrature-phase stationary noise}

We now turn to consider some nontrivial consequences of the quantum Langevin equations derived above [Eqs. $(2.22 a)$ and $(2.22 b)]$, which may be written in compact vector form:

$$
\frac{d}{d t} \underline{Z}=\underline{A Z}(t)+\Gamma(t)
$$

where $\underline{Z}(t)=\left(Z_{1}, Z_{2}, Z_{1}^{\dagger}, Z_{2}^{\dagger}\right)^{T}$ and the $4 \times 4$ drift and diffusion matrices are given in block form by

$$
\begin{aligned}
& \underline{A}=\left(\begin{array}{cc}
\mathcal{A} & 0 \\
0 & \mathcal{A}^{*}
\end{array}\right], \\
& \mathcal{A}(\sigma)=\left(\begin{array}{cc}
-a+b & 1 / 2 i c \\
-1 / 2 i c & -a-b
\end{array}\right)
\end{aligned}
$$

with

$$
\begin{aligned}
& a=\gamma\left(1+\frac{q}{2}\right), \\
& b=\sigma \gamma \sqrt{1+q}, \\
& c=\gamma q,
\end{aligned}
$$

and $\underline{D}=\underline{D}^{\mathrm{nsq}}+\underline{D}^{\mathrm{sq}}$ as above [see Eqs. (2.24) and (2.25)].

Note that the same formal Langevin equation allows us to take into account the two physical settings of our parametric amplifier device: the first one corresponding to the ordinary setup with nonsqueezed-vacuum fluctuations being amplified (this corresponds to taking $q=0$ in the previous equations), while the second one corresponds to an additional squeezed vacuum being injected into the cavity through a second transmitting mirror $(q>0)$.

To begin with we derive from the quantum Langevin Eqs. (3c) and (3d) the equation of motion for the $4 \times 4$ complex covariance matrix:

$$
\underline{C}(t)=\left\langle\underline{Z}(t) \underline{Z}(t)^{T}\right\rangle .
$$

Following the usual procedure $[3,11]$ of noise operator theory, a generalized Einstein relation is derived:

$$
\frac{d}{d t} \underline{C}(t)=\underline{A C}(t)+\underline{C}(t) \underline{A}+\underline{D} .
$$

The stationary covariances for the intracavity quadrature-phase-amplitude operators may now be obtained straightforwardly from this equation, by setting the left-hand side in Eq. (3.7) equal to zero and solving the corresponding matrix equation. Restricting ourselves to the squeezed quadrature (corresponding to the smallest negative eigenvalue of the drift matrix) we are led to the following covariances:

$$
\begin{aligned}
\left\langle Z_{2}^{\dagger} Z_{2}\right\rangle_{s s} & =\frac{1}{2}\left(\left\langle a_{+}^{\dagger} a_{+}\right\rangle_{s s}+\left\langle a_{-} a_{-}^{\dagger}\right\rangle_{s s}-2 \operatorname{Re}\left\langle a_{-} a_{+}\right\rangle_{s s}\right) \\
& =\frac{1}{2} \frac{1+q(1+N)}{1+q / 2} \frac{1+q / 2-\sigma \sqrt{1+q}}{1+q-\sigma^{2}(1+q)}
\end{aligned}
$$

The behavior of this covariance is shown in Fig. 2. It is to be noticed that a true divergence exists at threshold $(\sigma \rightarrow 1)$ for $q>0$. This is explained by the fact that the relaxation time of the quadrature-phase amplitude goes to infinity for $\sigma=1$. The corresponding fluctuations show a drastic slowing down in the vicinity of the critical point in contrast with the case for $q=0$ (no additional losses through a second mirror). The sharp region around the instability point should therefore be addressed by a proper nonlinear theory.

The condition for nondegenerate squeezing may now be stated in terms of the symmetrized covariance:

$$
\frac{1}{2}\left(\left\langle Z_{2} Z_{2}^{\dagger}\right\rangle+\left\langle Z_{2}^{\dagger} Z_{2}\right\rangle\right)_{s s}<\frac{1}{2} .
$$

From Eq. (3.8) and noticing that $\left\langle Z_{2} Z_{2}^{\dagger}\right\rangle=\left\langle Z_{2}^{\dagger} Z_{2}\right\rangle$ under the assumed approximation $v / \Omega \approx 0$, results the following condition for $q \ll<1$ results:

$$
\frac{1+[q /(1+q)] N}{2(1+\sigma)}<\frac{1}{2},
$$

which is to be contrasted with the case for $q=0$, but thermal noise entering the cavity through mirror $C$ :

$$
\frac{(1+2 \bar{n})}{2(1+\sigma)}<\frac{1}{2}
$$

the last inequality being satisfied for sufficiently low thermal noise $(2 \bar{n}<\sigma)$. According to Eq. (3.13) the limit

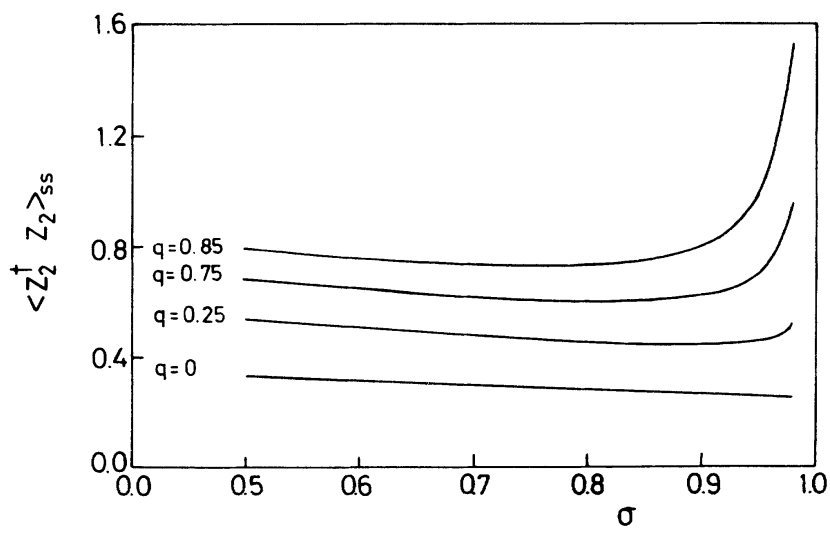

FIG. 2. Behavior vs the pumping parameter $\sigma$ of the stationary covariance [Eq. (3.8)] for different values of the transmittivity ratio $q$ and $r=1$. 
0.25 near threshold is obtained for the squeezed quadrature covariance (for $\bar{n}=0$ ), in accordance with previous work [20]. Input broadband squeezed noise [whose spectral density is measured by the parameter $\left.N=\sinh (r)^{2}\right]$ behaves in the first case much as thermal noise does in the ordinary one, degrading the level of available squeezing. As a numerical example, for $q=0.1$ and $\sigma=0.75$, the quadrature noise is lower than 0.40 for $r<1.5$ $(N<4.5)$.

The squeezed two-mode vacuum generated in the stationary state may be expressed in any case as a Gaussian state with this covariance. Nevertheless, a very important difference is apparent between the stationary squeezed states for $q=0$ and $q>0$. For $q=0$, the vacuum state generated has the null covariances $\left\langle\boldsymbol{Z}_{k} \boldsymbol{Z}_{m}\right\rangle=0$ $(k, m=1,2)$. This means that the noise in the quadrature phases is distributed randomly in phase (time stationary noise in the quadrature phases). On the contrary, for $q>0$, we have

$$
\begin{aligned}
\left\langle Z_{2} Z_{2}\right\rangle_{s s}=- & \frac{1}{2} \frac{1}{1+q / 2} \frac{1+q / 2-\sigma \sqrt{1+q}}{1+q-\sigma^{2}(1+q)} \\
& \times q M \exp (-2 i \phi)
\end{aligned}
$$

which clearly shows phase-sensitive noise in the two orthogonal Hermitian components of the (non-Hermitian)

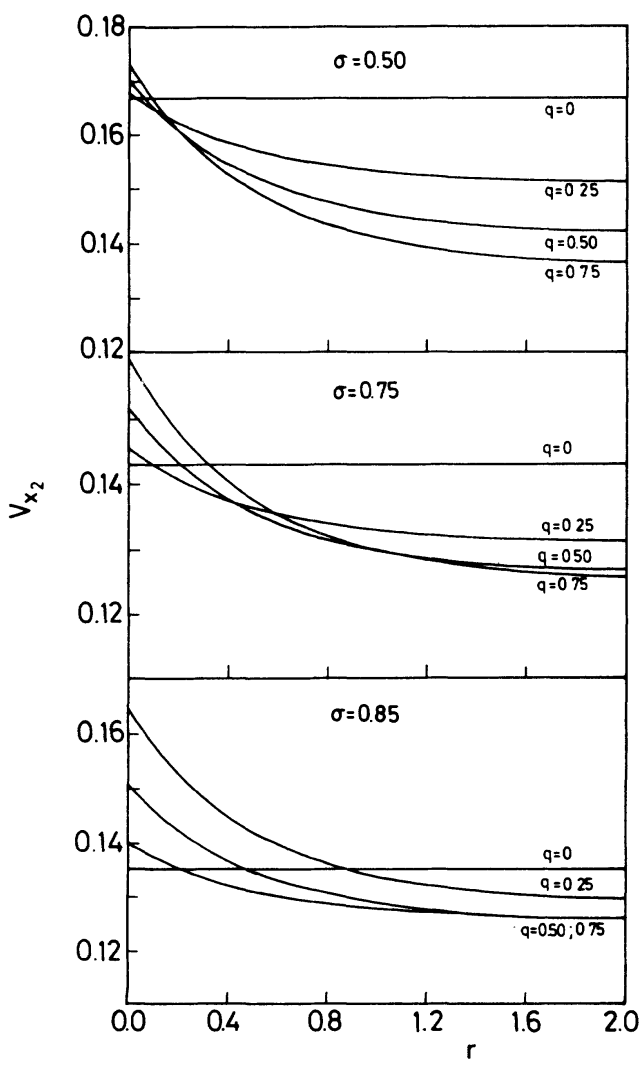

FIG. 3. Behavior of the stationary variances [Eq. (3.13)] vs the input squeezing parameter $r$ for three different values of the pumping parameter $\sigma$. The three sets of curves correspond to the transmittivity ratios displayed in the figure. quadrature-phase amplitude $Z_{2}$. In fact if we write

$$
Z_{2}=X_{2}+i Y_{2}
$$

the two Hermitian components of the squeezed quadrature (commutator $\left[X_{2}, Y_{2}\right]=i v / 2 \Omega \approx 0$ ) carry phasesensitive fluctuations. For an ordinary two-mode coherent or squeezed state $\left\langle X_{2} X_{2}\right\rangle=\left\langle Y_{2} Y_{2}\right\rangle \leq \frac{1}{4}$. Now from the stationary covariances, Eqs. (3.8) and (3.12), it results in

$$
\begin{aligned}
\left\langle X_{2} X_{2}\right\rangle_{s s} & =\frac{1}{2}\left(\left\langle Z_{2}^{\dagger} Z_{2}\right\rangle_{\text {sym }}+\operatorname{Re}\left\langle Z_{2} Z_{2}\right\rangle\right) \\
& =\frac{1}{4} \frac{1}{1+q / 2} F(\sigma)[1+q+q(N-M \cos (2 \phi)]
\end{aligned}
$$

where we have written $F(\sigma)=(1+q / 2-\sigma \sqrt{1+q}) /$ $\left[1+q-\sigma^{2}(1+q)\right]$. For $\phi=0$ and a certain range of the other parameters, $\left\langle X_{2} X_{2}\right\rangle_{s s}$ may reach lower values than 0.25 , manifesting as a quadrature-squeezed state, i.e., time-dependent nonstationary noise in the quadrature phases of the field [10]. Some representative graphs for several values of $\sigma$ are depicted in Fig. 3.

\section{INPUT-OUTPUT CORRELATIONS: THE OUTPUT SPECTRUM OF SQUEEZING}

\author{
A. Relation between input, output, \\ and intracavity fields: The output spectrum
}

This model of a two-mode coupled oscillator interacting with a bath is the simplest model for a nondegenerate parametric amplifier within a resonant cavity. As it has been previously noted, there is a need for a supplementary theory to relate the internal squeezing of the discrete resonant modes within the cavity with the actual squeezing measured in the multimode output-cavity field [21]. In this section we derive the special structure of a squeezed field output spectrum as it is obtained within the quadrature-phase formalism outlined above. For the sake of clarity we discuss independently the two cases worked out in previous sections (ordinary vacuum noise or squeezed noise entering the cavity through one or two mirrors, see Secs. II and III). We assume thus at first, for the analysis of the output spectrum of quadrature fluctuations, the physical configuration of a ring cavity system coupled with the outside world through a single transmitting mirror.

Following Collett and Gardiner and Carmichael [21], we can relate the output field to the internal field and doing so, identify the physical origin of the Langevin noise sources described above. Let us write the output (linearly polarized) electric field operator in the form

$$
E(z, t)=E^{(+)}(z, t)+E^{(-)}(z, t)
$$

where the usual decomposition into positive- and negative-frequency components has been made.

The positive-frequency part may be expressed in terms of the bath creation-annihilation operators: 


$$
\begin{aligned}
E^{(+)}(z, t)= & \sum_{\alpha}\left(\frac{\hbar \omega_{1 \alpha}}{2 \epsilon_{0} A L}\right)^{1 / 2} c_{1 \alpha}(t) \exp \left(i \omega_{1 \alpha} z / c\right) \\
& +\sum_{\alpha}\left(\frac{\hbar \omega_{2 \alpha}}{2 \epsilon_{0} A L}\right)^{1 / 2} c_{2 \alpha}(t) \exp \left(i \omega_{2 \alpha} z / c\right)
\end{aligned}
$$

The Heisenberg operators $c_{1 \alpha}(t)$ and $c_{2 \alpha}(t)$ may be readily integrated from the exact Heisenberg system-bath equations. When substituted into the last equation we obtain

$$
E^{(+)}(z, t)=E_{\text {free }}^{(+)}(z, t)+E_{\text {source }}^{(+)}(z, t)
$$

where the first term only depends upon the initial state of the bath and the "source" term is related to the intracavity system operators. When the standard approximations are performed (continuous distribution of bath frequencies, Markovian limit), we obtain for the "source" term the following expression:

$$
\begin{aligned}
E_{\text {source }}^{(+)}(z, t)= & \sqrt{2 \gamma} \exp [-i \Omega(t-z / c)] \\
\times & {\left[\left(\frac{\hbar(\Omega+v)}{2 c \epsilon_{0} A}\right]^{1 / 2} \widetilde{a}_{+}(t-z / c)\right.} \\
& \left.+\left(\frac{\hbar(\Omega-v)}{2 c \epsilon_{0} A}\right]^{1 / 2} \widetilde{a}_{-}(t-z / c)\right]
\end{aligned}
$$

valid for $c t>z \geq 0$. Following Caves and Schumaker we rescale the electric field in photon units as $\mathscr{E}^{(+)}(z, t)=\left(2 \epsilon_{0} c A / \hbar \Omega\right)^{1 / 2} E^{(+)}(z, t)$. Then we obtain from Eq.(4.2)

$$
\begin{aligned}
& \mathscr{E}_{\text {source }}^{(+)}(z, t)= \exp [-i \Omega(t-z / c)] 2 \sqrt{\gamma} \\
&\left.\times\left[\lambda_{+} \widetilde{a}_{+}(t-z / c)+\lambda_{-} \widetilde{a}_{-}(t-z) / c\right)\right] \\
& {\left[\lambda_{ \pm}=\sqrt{(\Omega+v) / 2 \Omega}\right] . }
\end{aligned}
$$

We can now identify the quadrature components of the measured output field. To do so it is sufficient to observe that the measured field at any time may be considered to propagate freely from the field at the output mirror of the cavity ( $z=0$ coordinate) so that we may write the observed quadrature fields in the form

$$
\mathscr{E}_{m}^{\text {out }}(t)=\mathscr{E}_{m}^{\text {in }}(t)+2 \sqrt{\gamma}\left[\widetilde{\boldsymbol{Z}}_{m}(t)+\widetilde{Z}_{m}^{\dagger}(t)\right]
$$

so that $\mathscr{E}(t)=\mathscr{E}_{1}^{\text {out }}(t) \cos (\Omega t)+\mathscr{E}_{2}^{\text {out }}(t) \sin (\Omega t)$ is the measured real electric field in photon units. $\mathscr{E}_{m}^{\text {in }}(t)$ may be easily interpreted as the quadrature components of the vacuum fields propagating into the cavity from the outcavity environment and reflecting simultaneously onto the cavity output (see Ref. [21]).

The Langevin quantum sources of noise in Eqs. (2.8) may now be easily related to the input quadrature (Hermitian) operators $\mathscr{E}_{m}^{\text {in }}(t)$ and the following relation holds:

$$
\widetilde{\Gamma}_{m}(t)+\widetilde{\Gamma}_{m}^{\dagger}(t)=-\sqrt{\gamma} \mathscr{E}_{m}^{\text {in }}(t)
$$

where

$$
\widetilde{\Gamma}_{m}(t)=\exp (-i v t) \Gamma_{m}(t) .
$$

Equations (4.6)-(4.8) will now be the point of departure for the analysis of the output squeezing spectrum. We define the quadrature fluctuation spectrum by means of $V_{m}(\omega)(m=1,2)$, defined by

$$
V_{m}(\omega)=\int_{0}^{\infty}\left\langle\mathscr{E}_{m}(t) \mathscr{E}_{m}(t+\tau)\right\rangle_{\mathrm{sym}}^{\text {out }} \cos \omega \tau d \tau
$$

As is well known, when heterodyne detection of the combination of the two side-band modes is performed, the photocurrent fluctuation spectrum has Fourier components just proportional to

$$
V(\theta, \omega)=\int_{0}^{\infty}\left\langle\mathscr{E}^{\mathrm{out}}(\theta, t) \mathscr{E}^{\mathrm{out}}(\theta, t+\tau)\right\rangle_{\mathrm{sym}} \cos \omega \tau d \tau
$$

where $\mathscr{E}^{\text {out }}(\theta, t)=\cos \theta \mathscr{E}_{1}^{\text {out }}(t)+\sin \theta \mathscr{E}_{2}^{\text {out }}(t)$, the noise in each "rotated" quadrature $\mathscr{E}^{\text {out }}(\theta, t)$ being detected by a suitably chosen local oscillator phase [10]. The maximum reduction of quadrature-phase noise corresponds to that quadrature maximally deamplified as may be seen from the Langevin Eqs. (2.8a) and (2.8b). Therefore it will be sufficient to limit our analysis to the $Z_{2}(\theta=\pi / 2)$ quadrature-phase noise. Taking into account Eq. (4.6) we obtain the following structure for the spectral density:

$$
V(\pi / 2, \omega)=V_{2}(\omega)=V^{\text {in }}(\omega)+V^{\text {cavity }}(\omega)+V^{\text {correl }}(\omega)
$$

where

$V^{\text {in }}(\omega)=\int_{0}^{\infty}\left\langle\mathscr{E}_{2}(t) \mathscr{E}_{2}(t+\tau)\right\rangle_{\text {sym }}^{\text {in }} \cos \omega \tau d \tau$

Here $V^{\text {in }}(\omega)$ arises from the quadrature-phase noise carried by the vacuum field. This just amounts to the phase invariant value of $\frac{1}{2}$ and measures the ordinary quantum limit for the coherent-vacuum state. The second term is related to the intracavity quadrature spectrum:

$V^{\text {cavity }}(\omega)=4 \gamma \int_{0}^{\infty}\left[\left\langle\widetilde{Z}_{2}(t) \widetilde{Z}_{2}(t+\tau)\right\rangle_{\text {sym }}+\left\langle\widetilde{\boldsymbol{Z}}_{2}^{\dagger}(t) \widetilde{\boldsymbol{Z}}_{2}^{\dagger}(t+\tau)\right\rangle_{\mathrm{sym}}+\left\langle\widetilde{\boldsymbol{Z}}_{2}(t) \widetilde{\boldsymbol{Z}}_{2}^{\dagger}(t+\tau)\right\rangle_{\mathrm{sym}}+\left\langle\widetilde{\boldsymbol{Z}}_{2}^{\dagger}(t) \widetilde{\boldsymbol{Z}}_{2}(t+\tau)\right\rangle_{\mathrm{sym}}\right] \cos \omega \tau d \tau$

As is shown below, this spectral term has positive values for all frequencies. The third term in Eq. (4.11) is therefore responsible for the observed output squeezing at a suitable range of frequencies. As follows from Eqs. (4.6)-(4.9), $V^{\text {correl }}(\omega)$ arises from correlations between the free-quadrature fields and the intracavity system and is given by 


$$
\begin{aligned}
V^{\text {correl }}(\omega)=2 \sqrt{\gamma} \int_{0}^{\infty}[ & \left\langle\mathscr{E}_{2}^{\text {in }}(t) \widetilde{Z}_{2}(t+\tau)\right\rangle_{\text {sym }}+\left\langle\mathscr{E}_{2}^{\text {in }}(t) \widetilde{Z}_{2}^{\dagger}(t+\tau)\right\rangle_{\text {sym }} \\
& \left.+\left\langle\widetilde{Z}_{2}(t) \mathscr{E}_{2}^{\text {in }}(t+\tau)\right\rangle_{\text {sym }}+\left\langle\widetilde{Z}_{2}^{\dagger}(t) \mathscr{E}_{2}^{\text {in }}(t+\tau)\right\rangle_{\text {sym }}\right] \cos \omega \tau d \tau .
\end{aligned}
$$

Going further with the calculation of $V^{\text {cavity }}(\omega)$, we first define the spectral matrix:

$$
\underline{S}(\omega)=\int_{-\infty}^{+\infty}\left\langle\underline{Z}(t) \underline{Z}(t+\tau)^{T}\right\rangle \exp (-i \omega \tau) d \tau
$$

where $\underline{Z}(t)=\left(Z_{1}, Z_{2}, Z_{1}^{\dagger}, Z_{2}^{\dagger}\right)^{T}$. It is straightforward to write $V^{\text {cavity }}(\omega)$ in terms of the following matrix elements of $S(\omega)$ :

$$
\begin{aligned}
V^{\text {cavity }}(\omega)=(4 \gamma / 2)[ & \frac{1}{2} S_{22}(\omega+v)+\frac{1}{2} S_{22}(-\omega+v)+\frac{1}{2} S_{44}(\omega-v)+\frac{1}{2} S_{44}(-\omega-v) \\
& \left.+\frac{1}{2} S_{42}(\omega+v)+\frac{1}{2} S_{24}(\omega-v)+\frac{1}{2} S_{42}(-\omega+v)+\frac{1}{2} S_{24}(-\omega-v)\right]
\end{aligned}
$$

where we have taken into account that $\exp (i v t) Z_{i}(t)=\exp (i v t) \widetilde{Z}_{i}(t)$.

Equations (4.13) and (4.7) allow us to write $V^{\text {correl }}(\omega)$ in terms of the correlations between intracavity quadraturephase amplitudes and the Langevin-noise operators $\Gamma_{i}(t)$. For that purpose we further define the spectral matrices:

$$
\begin{aligned}
& \underline{H}(\omega)=\int_{-\infty}^{+\infty}\left\langle\Gamma(0) \underline{Z}(\tau)^{T}\right\rangle \exp (-i \omega \tau) d \tau, \\
& \underline{G}(\omega)=\int_{-\infty}^{+\infty}\left\langle\underline{Z}(\tau) \Gamma(0)^{T}\right\rangle \exp (-i \omega \tau) d \tau .
\end{aligned}
$$

Substituting $\mathscr{E}_{m}^{\text {in }}(t)$ from Eq. (4.7) into Eq. (4.14), and making use of the time invariance of the stationary two-time correlation functions:

$$
\left\langle\Gamma(-\tau) \underline{Z}(0)^{T}\right\rangle=\left\langle\Gamma(0) \underline{Z}(\tau)^{T}\right\rangle
$$

for $\tau>0$, we can easily relate $V^{\text {correl }}(\omega)$ to certain matrix elements of $\underline{H}(\omega)$ and $\underline{G}(\omega)$. Proceeding as above we are led to the following expression:

$$
\begin{aligned}
V^{\text {correl }}(\omega)=-\frac{1}{2}[ & G_{22}(\omega+v)+G_{42}(\omega+v)+G_{22}(-\omega+v)+G_{42}(-\omega+v) \\
& +H_{22}(\omega+v)+H_{24}(\omega+v)+H_{22}(-\omega+v)+H_{24}(-\omega+v) \\
& +G_{24}(\omega-v)+G_{44}(\omega-v)+G_{24}(-\omega-v)+G_{44}(-\omega-v) \\
& \left.+H_{42}(\omega-v)+H_{44}(\omega-v)+H_{42}(-\omega-v)+H_{44}(-\omega-v)\right] .
\end{aligned}
$$

The complete form of the output squeezing spectrum may then be obtained by means of the elements of the three spectral matrices defined above [Eqs. (4.14)-(4.16)]. The form of these matrices may now be made explicit as the following formulas may readily be derived making use of the quantum-regression theorem [3]:

$$
\begin{aligned}
& \underline{G}(\omega)=\underline{D}\left(i \omega-\underline{A}^{T}\right)^{-1}, \\
& \underline{H}(\omega)=(i \omega-\underline{A})^{-1} \underline{D}, \\
& \underline{S}(\omega)=(A+i \omega)^{-1} \underline{D}\left(\underline{A}^{T}-i \omega\right)^{-1} .
\end{aligned}
$$

We may then calculate these spectral matrices from the explicit form of the drift and diffusion matrices which may be immediately written from Eqs. (2.8) and (2.9) in Sec. II A, for the case of a reservoir field in an ordinary vacuum state.

As it may be seen from Eq. (4.22), the first four terms in the right-hand side of Eq. (4.16) stem from the phase sensitivity of the noise present in each of the intracavity quadrature fields. As it is shown in $[9,10]$, this is a null contribution if the intracavity signal has time-stationaryquadrature-phase (TSQP) noise, and this is in fact the case for the present setting of the linear parametric amplifier. Straightforward algebra leads us to the following terms:

$$
\begin{aligned}
& S_{22}(\omega)=S_{44}(\omega)=0, \\
& G_{22}(\omega)=G_{44}(\omega)=H_{22}(\omega)=H_{44}(\omega)=0 .
\end{aligned}
$$

Further properties will be discussed below for the general expression of Eq. (4.19).

Noting that $\underline{A}$ is a diagonal matrix in the present case, the calculation of the relevant matrix elements is straightforward and it results in the following simple expression for the spectrum of quadrature squeezing:

$$
\begin{aligned}
V_{2}(\omega)= & V^{\text {in }}(\omega)+V^{\text {cavity }}(\omega)+V^{\text {correl }}(\omega) \\
= & \frac{1}{2}+2 \gamma \frac{\gamma}{(\omega-v)^{2}+\gamma^{2}(1+\sigma)^{2}}+2 \gamma \frac{\gamma}{(-\omega-v)^{2}+\gamma^{2}(1+\sigma)^{2}} \\
& -\gamma\left(\frac{1}{i(\omega-v)+\gamma(1+\sigma)}+\frac{1}{i(-\omega-v)+\gamma(1+\sigma)}+\text { c.c. }\right) .
\end{aligned}
$$


If the intermode frequency separation is much greater than the cavity linewidth, the squeezing spectrum for the maximally squeezed quadrature may be well approximated in the vicinity of $\omega=v$, with the following results:

$$
V_{2}(\omega)=\frac{1}{2}-\frac{2 \sigma \gamma^{2}}{(\omega-v)^{2}+\gamma^{2}(1+\sigma)^{2}} .
$$

As may be immediately seen from the last expression, this formula leads to $V_{2}(\omega)=0$ (100\% noise reduction) for $\omega=v$, in the limit $\sigma \rightarrow 1$ (threshold point for oscillation). This is a well-known result for ideal parametric two-photon devices [21].

\section{B. Output quadrature spectrum: Phase-sensitive squeezed reservoir}

The output spectrum of quadrature-phase fluctuations (squeezing spectrum) will now be obtained for the cavity amplifier when an additional input reservoir field with broadband squeezed white noise enters the cavity as discussed before. We shall proceed, with minor changes, along the same lines as in the preceding section.

The relationship between input and output quadrature fields is formally unchanged [cf. Eq. (4.6)] and we write it for clarity:

$\mathscr{E}_{m}^{\text {out }}(t)=\mathscr{E}_{c, m}^{\text {in }}(t)+2 \sqrt{\gamma}\left[\widetilde{Z}_{m}(t)+\widetilde{Z}_{m}^{\dagger}(t)\right] \quad(m=1,2)$

where now the first term stems from the vacuum quadrature noise entering the cavity through mirror $C$.

In addition the input quadrature field is related to the Langevin operators, as follows immediately from Eqs. (2.23) and (2.23b):

$$
\widehat{\Gamma}_{m}^{\mathrm{nsq}}(t)+\widetilde{\Gamma}_{m}^{\mathrm{nsq}}{ }^{\dagger}(t)=-\sqrt{\gamma} \mathscr{E}_{c, m}^{\mathrm{in}}(t)
$$

where $\hat{\Gamma}_{m}^{\text {nsq }}(t)$ refers to that part of the Langvin forces related to the nonsqueezed noise transmitted into the cavity through mirror $C$.

The fluctuation spectrum in each of the quadratures of the output field may now be easily calculated. Proceeding along the same lines as above, we are led to the following quadrature spectrum:

$$
V_{2}(\omega)=V^{\text {in }}(\omega)+V^{\text {cavity }}(\omega)+V^{\text {correl }}(\omega)
$$

where the three spectral contributions are expressed with some minor changes as done in Eqs. (4.11)-(4.13). We use again the spectral matrix $\underline{S}(\omega)$ defined by Eq. (4.14), and substitute $\Gamma$ by $\Gamma^{\mathrm{nsq}}$ in the expressions for $\underline{G}(\omega)$ and $\underline{H}(\omega)$ as defined by Eqs. (4.16) and (4.17).

The formulas given in Eqs. (4.20) and (4.21) are still valid, with the $4 \times 4$ diffusion matrix replaced by $\underline{D}^{\text {nsq }}$ [Eq. (2.25)] and the drift matrix written in block-diagonal form in Eqs. (3.5a) and (3.5b) [cf. also Eqs. (2.22a) and (2.22b)].

We note in turn that $\underline{D}^{\text {nsq }}[$ (see Eq. (2.25)] is an Hermitian matrix, so the following relation between $\underline{G}$ and $\underline{H}$ holds:

$$
\underline{H}(\omega)=[\underline{G}(-\omega)]^{\dagger} .
$$

A straightforward but lengthy calculation leads to the following final spectral terms for the output squeezing spectrum in the output quadrature corresponding to the most strongly damped intracavity quadrature:

$S_{42}(\omega)=\frac{\gamma[1+q(1+N)]}{\mathcal{L}(\omega)}\left[(a-b)^{2}+\omega^{2}+\frac{1}{4} c^{2}-c(a-b)\right]$,

$S_{24}(\omega)=\frac{\gamma}{\mathcal{L}(\omega)}\left\{(1+q N)\left[(a-b)^{2}+\frac{1}{4} c^{2}+\omega^{2}\right]\right.$

$$
+c(1-q N)(a-b)\},
$$

$S_{44}(\omega)=S_{22}(-\omega)^{*}$

$$
\begin{aligned}
= & \frac{-q \gamma}{\mathcal{L}(\omega)} M \exp (2 i \phi) \\
& \times\left[(a-b)^{2}+\omega^{2}+\frac{1}{4} c^{2}-c(a-b)\right],
\end{aligned}
$$

$$
\begin{aligned}
G_{24}(\omega)= & \frac{\gamma}{\mathcal{L}(\omega)}\left(a^{2}-b^{2}-\frac{1}{4} c^{2}-\omega^{2}-2 a i \omega\right) \\
& \times\left[\frac{c}{2}+(a-b)+i \omega\right], \\
H_{42}(\omega)= & -\frac{\gamma}{\mathcal{L}(\omega)}\left(a^{2}-b^{2}-\frac{1}{4} c^{2}-\omega^{2}-2 a i \omega\right) \\
& \times\left(\frac{c}{2}-(a-b)-i \omega\right)
\end{aligned}
$$

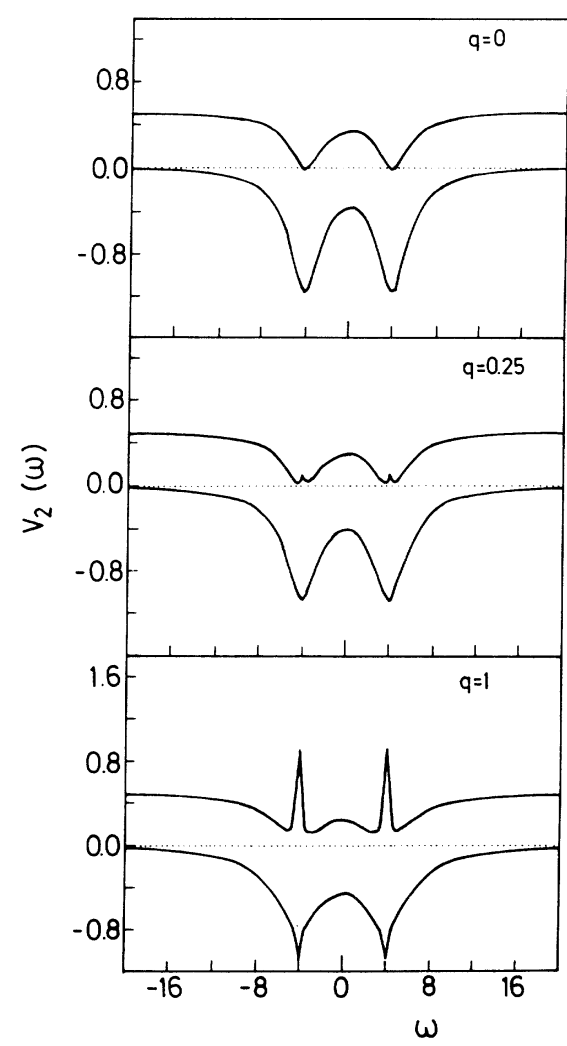

FIG. 4. Spectra of quadrature squeezing for three different transmittivities. The upper trace corresponds to $V_{2}(\omega)$ and the lower ones to the correlation component $V_{\text {correl }}(\omega)$. All of the spectra correspond to the values of $r=1, \sigma=0.8, v=4, \phi=0$. 


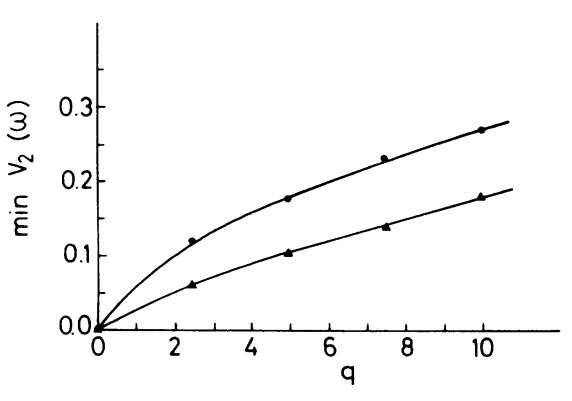

FIG. 5. A comparison between the maximally attainable squeezing when coherent- (dots, $r=0$ ) or squeezed- (triangles, $r>0$ ) vacuum inputs enter through mirror $d$ for several values of the transmittivity ratio $q$.

where we have written

$$
\mathcal{L}(\omega)=\left(a^{2}-b^{2}-\frac{1}{4} c^{2}-\omega^{2}\right)^{2}+4 a^{2} \omega^{2} .
$$

Some representative spectra for a selected set of parameter values are displayed in Fig. 4. As can be seen upon inspection of the graphs, the out-of-cavity spectrum which is constituted by the sum of the three contributions given by Eq. (4.28) shows reduced fluctuations around the central frequency and a region of quadrature-squeezed noise below the input level for a range of parameters. Such a reduction is mainly caused by the correlation term $V^{\text {correl }}(\omega)$, whereas the cavity contribution $V^{\text {cavity }}(\omega)$ counteropposes this effect since it is a positive quantity in all the frequency range.

As an example Fig. 5 shows a comparison of the maximum squeezing attainable for coherent-vacuum and squeezed-vacuum inputs, respectively, for several values of the transmittivity ratio $q$. It can be clearly seen upon inspection of the figure that for nonzero values of $q$ the input of a squeezed vacuum substantially compensates the deleterious effect of the added transmission losses. Finally, it is to be noticed that the appearance of a central structure (for the $q>0$ spectra) signals the instability near the threshold already mentioned above (see Sec. III).

\section{DISCUSSION}

The dynamics of a well-known nonlinear-optical device currently used for the generation of squeezed states of light has been reformulated by means of the quadraturephase-amplitude formalism. The relevance of such an analysis stems from the following facts. First of all, fully classical analogues are easily found which are free from the well-known effects introduced by the use of standard, positive- $P$ representation methods, that is, the appearance of nonpositive-definite diffusion matrices. Although it has been argued that such negative diffusion phenomena were a true manifestation of the quantum character of the dynamics, it seems nowadays clear that such a difficulty can be circumvented using a different representation.

On the other hand, the advantage of having a clear classical counterpart in the linear subthreshold regime of two-photon optical devices enables the use of computersimulation techniques for the more complicated nonlinear above-threshold problems, a technique that was rather difficult to use in the previous positive- $P$ version of the formalism. In this respect, it is worth noticing that, although some linearization procedure should be applied for problems involving higher than quadratic interactions, very useful results can be obtained provided that the calculations are carried out far from critical points (i.e., threshold).

A number of optical two-mode devices may also be readily analyzed along the lines described above. In particular, we mention the recent studies on resonancefluorescence models directed to the squeezed-state generation [22] and four-wave mixing nondegenerate amplifiers with a two-photon decay mechanism [23] as some problems which can be reformulated using the method proposed in this work. In such cases it may be interesting to analyze the out-of-cavity spectrum of squeezing along the same lines sketched in Sec. IV of the present paper, since something may be gained from the separation of noise sources into different contributions as proposed above. This may be especially relevant when phase-dependent broadband squeezed reservoirs are included.

Finally, it seems clear that a substantial effort should be directed towards tackling complicated nonlinear problems in a search for clear classical analogues. At the present time only the first steps [24] towards this goal have been taken and further efforts are required.

\section{ACKNOWLEDGMENTS}

Work supported in part by Grants No. TIC $90-80$, from DGICYT (Spain), and No. A-91002002-15, from U.P.M. (Madrid, Spain). Professor M. San Miguel is acknowledged for encouraging support and stimulating discussions.
[1] L. Knöll, W. Vogel, and D. G. Welsch, Phys. Rev. A 43, 543 (1991); J. Gea Banacloche et al., ibid. 41, 369 (1990).

[2] W. H. Louisell, Quantum Statistical Properties of Radiation (Wiley, New York, 1973).

[3] P. Meystre and M. Sargent III, Elements of Quantum Optics (Springer-Verlag, Berlin, 1990), especially Chap. 14.

[4] C. Cohen-Tannoudji, J. Dupont-Roc, and G. Grynberg, Introduction à l'Electrodynamique Quantique (CNRS, Paris, 1988).
[5] H. Haken, Light (North-Holland Amsterdam, 1981), Vol. II.

[6] See, for example, P. Mestre and M. Sargent III, Ref. [3], and references therein.

[7] P. D. Drummond and C. W. Gardiner, J. Phys. A 13, 2353 (1980).

[8] T. W. Marshall and E. Santos, Phys. Rev. A 41, 1582 (1990).

[9] C. M. Caves and B. L. Schumaker, Phys. Rev. A 31, 3068 
(1985); C. M. Caves, Phys. Rev. D 26, 1817 (1982).

[10] C. M. Caves and B. L. Schumaker, in Quantum Optics IV, edited by J. D. Harvey and D. F. Walls (Springer-Verlag, Berlin, 1986), p. 20.

[11] For the quantum-Langevin equations of a system coupled to a reservoir see especially Chap. IV in C. CohenTannoudji, J. Dupont-Roc, and G. Grynberg, Ref. [4] and also M. Lax, Phys. Rev. 145, 110 (1966).

[12] R. Graham, Phys. Rev. Lett. 52, 117 (1984).

[13] For a very illuminating discussion of quantum versus classical squeezing of noise, see N. B. Abraham, in Dynamics of Non-Linear Optical Systems, edited by L. Pesquera and F. J. Bermejo (World Scientific, Singapore, 1989), p. 29, and references therein.

[14] H. J. Carmichael, A. S. Lane, and D. F. Walls, Phys. Rev. Lett. 28, 2539 (1987).

[15] C. W. Gardiner, Phys. Rev. Lett. 56, 1917 (1986).

[16] A. K. Ekert and P. L. Knight, Phys. Rev. A 42, 487 (1990).
[17] C. W. Gardiner and M. J. Collet, Phys. Rev. A 31, 3761 (1985).

[18] For a complete analysis of the nondissipative parametric two-mode amplifier with the use of the Wigner function, see B. R. Mollow and R. J. Glauber, Phys. Rev. 160, 1097 (1967).

[19] M. Lax, Phys. Rev. 172, 350 (1968).

[20] B. L. Schumaker and C. Caves, in Coherence and Quantum Optics V, edited by L. Mandel and E. Wolf (Plenum, New York, 1984), p. 743.

[21] M. J. Collet and D. F. Walls, Phys. Rev. A 32, 2887 (1985); M. J. Collet and C. W. Gardiner, ibid. 30, 1386 (1984); H. J. Carmichael, J. Opt. Soc. Am. B 4, 1588 (1987); M. J. Collet and R. Loudon, ibid. 4, 1525 (1987).

[22] T. Quang, Phys. Rev. A 41, 6313 (1990).

[23] N. Lu, Phys. Rev. A 41, 6261 (1990).

[24] G. Garcia Calderon and M. Moshinsky, J. Phys. A 13, L185 (1980). 